\title{
All-fiber high-power linearly polarized supercontinuum generation from polarization-maintaining photonic crystal fibers
}

\author{
Yue Tao ${ }^{1,2,3, \dagger}$ and Sheng-Ping Chen ${ }^{1,2,3, \dagger}$ \\ ${ }^{1}$ College of Advanced Interdisciplinary Studies, National University of Defense Technology, Changsha 410073, China \\ ${ }^{2}$ State Key Laboratory of Pulsed Power Laser Technology, Changsha 410073, China \\ ${ }^{3}$ Hunan Provincial Key Laboratory of High Energy Laser Technology, Changsha 410073, China \\ (Received 10 January 2019; revised 1 February 2019; accepted 14 March 2019)
}

\begin{abstract}
We demonstrate an all-fiber high-power linearly polarized supercontinuum source with polarization-maintaining photonic crystal fibers (PM-PCFs) as the nonlinear medium. The source exhibits an average output power of $3.8 \mathrm{~W}$ with a flat spectrum from $480 \mathrm{~nm}$ to $2100 \mathrm{~nm}$ at the $-10 \mathrm{~dB}$ level, except for the residual pump peak. The polarization extinction ratio (PER) is measured to be greater than $20 \mathrm{~dB}$ at selected sample wavelength points $(532 \mathrm{~nm}, 1064 \mathrm{~nm}$ and $1550 \mathrm{~nm}$ ) at the highest pump power level and greater than $20 \mathrm{~dB}$ at all wavelengths from $800 \mathrm{~nm}$ to $1500 \mathrm{~nm}$ at the low pump power level. We also experimentally study the spectral properties when the pump light propagates along different axes of the PM-PCF. The results show that propagating parallel to the slow axis enables a broader spectrum in the PM-PCF in this case, probably due to matching of the dispersion properties with the pump light, which is qualitatively in accordance with the numerical simulation. To our best knowledge, this is the first demonstration of a watt-level linearly polarized supercontinuum source generated from PM-PCFs in an all-fiber structure.
\end{abstract}

Keywords: linear polarization; photonic crystal fiber; polarization maintaining; supercontinuum

\section{Introduction}

Supercontinuum (SC) fiber sources have been a fruitful research area in recent years, and have the beneficial properties of a broadband spectrum, good spatial coherence and a compact structure for a wide range of applications, such as hyperspectral lidar, optical coherence tomography, optical frequency metrology, and white-light trapping ${ }^{[1-6]}$. The development of highly nonlinear photonic crystal fibers (PCFs) has greatly improved the interplay of multiple nonlinear effects for broadband SC generation ${ }^{[7,8]}$. By adjusting the geometrical parameters of a PCF, primarily including the core diameter, the air-hole diameter and the hole pitch, the dispersion properties, especially the zerodispersion wavelength (ZDW), can be designed to meet the phase-matching requirements, facilitating the realization of a broad SC spectrum ${ }^{[9-15]}$. The UV to near-IR region is important in biomedical imaging and fluorescence lifetime

Correspondence to: S.-P. Chen. College of Advanced Interdisciplinary Studies, National University of Defense Technology, Changsha 410073, China. Email: chespn@163.com

$\dagger$ These two authors contributed equally to this work. imaging fields ${ }^{[16,17]}$. However, these applications always require a linearly polarized $\mathrm{SC}$, whereas the generated $\mathrm{SC}$ is generally unpolarized.

At present, there are two methods for generating a linearly polarized SC. One is utilizing multiple nonlinear effects excited by a high peak power to extend the spectrum in a polarization-maintaining (PM) master oscillator power amplifier (MOPA) structure. Zhang et al. reported a $124 \mathrm{~W}$ linearly polarized SC in a PM MOPA system. The measured polarization extinction ratio (PER) of the whole SC source is about $7.5 \mathrm{~dB}^{[18]}$. Owing to the low nonlinear coefficient and the difficulty in controlling the dispersion properties of conventional fibers, the spectrum is broadened only from $850 \mathrm{~nm}$ to $1900 \mathrm{~nm}$. To further broaden the spectrum, highly nonlinear fibers such as PCFs have been widely used in broadband SC generation for their excellent characteristics, such as high nonlinearity and tailorable dispersion ${ }^{[19,20]}$. Therefore, another effective way for obtaining a linearly polarized SC is using highly birefringent $\mathrm{PCFs}^{[21-26]}$. In 2003, Lehtonen et al. realized SC broadening from 400 to $1750 \mathrm{~nm}$ in a $\mathrm{PCF}^{[27]}$. Tarnowski et al. demonstrated a polarized all-normal dispersion SC generated in a birefringent silica 


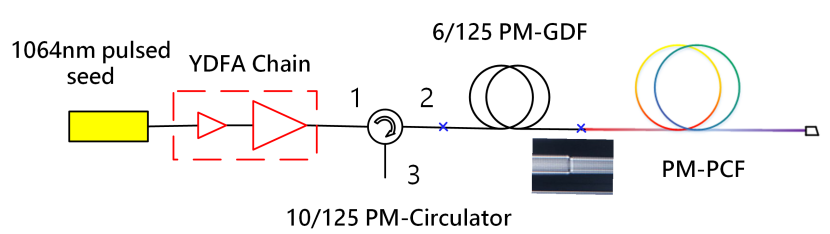

Figure 1. Experimental setup of the linearly polarized SC source.

PCF spanning from $750 \mathrm{~nm}$ to over $2500 \mathrm{~nm}$ in $2017^{[28]}$. These PCFs always have a noncircularly symmetric structure to induce high birefringence. The combination of high birefringence, dispersion and high nonlinearity allows the polarization state to be maintained while generating the SC. Also, the preserved polarization enhances the nonlinear interactions so that less power is required to generate the SC. However, most previously reported linearly polarized SC generation from PCFs has been spatially coupled, mainly limited by the severe mode field mismatch and the difficulty of polarization-maintaining alignment when splicing different kinds of fibers, and is often pumped with femtosecond pulses for high peak power to reach the nonlinear threshold. Therefore, the power of the linear polarization SC generated in PM-PCF is limited to the milliwatt level and the spectrum does not cover the whole visible to near-infrared region.

In this paper, we adopt a polarization-maintaining PCF splicing method and first demonstrate an all-fiber highpower linearly polarized SC source from PM-PCF. The PER is measured to be greater than $20 \mathrm{~dB}$ at selected sample wavelength points $(532 \mathrm{~nm}, 1064 \mathrm{~nm}$ and $1550 \mathrm{~nm})$ at the highest pump power level and greater than $20 \mathrm{~dB}$ at all wavelengths from $800 \mathrm{~nm}$ to $1500 \mathrm{~nm}$ at a low pump power level. Three different lengths of PM-PCFs $(1.6 \mathrm{~m}, 3 \mathrm{~m}$, and $6 \mathrm{~m}$ ) are used to study the effect of fiber length on SC generation. A different SC is generated when the pump pulses are polarized along different axes of the PMPCF. The experimental results are highly consistent with the theoretical simulation in terms of the spectral broadening tendency.

\section{Experimental setup}

The experimental configuration of the linearly polarized SC source is shown in Figure 1, comprising a picosecond pulsed seed source, an ytterbium-doped fiber amplifier (YDFA) chain with a pigtail of $10 / 125 \mu \mathrm{m}$ fiber, a $10 / 125 \mu \mathrm{m}$ polarization-maintaining circulator (PM-CIR), a piece of $0.2 \mathrm{~m}$ long $6 / 125 \mu \mathrm{m}$ germanium-doped fiber (PM-GDF), and a piece of PM-PCF. In our experiment, the seed and the YDFA chain act as the pump source to generate the SC, delivering $150 \mathrm{ps}$ duration pulses with a central wavelength of $1064 \mathrm{~nm}$ at a repetition rate of $4 \mathrm{MHz}$. The PER of the pump source is $10 \mathrm{~dB}$, monitored by an extinction ratio meter that operates in the wavelength range

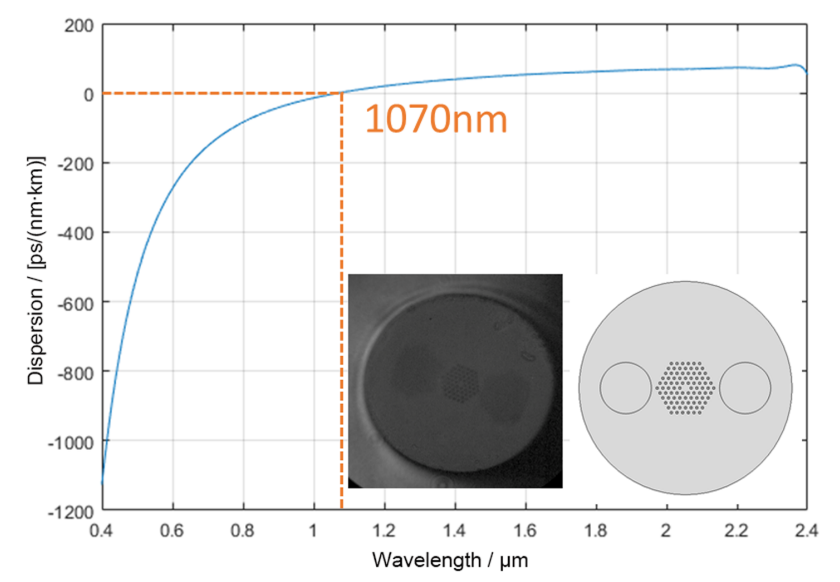

Figure 2. Calculated dispersion curve of the PM-PCF. Inset: micrograph cross-section and simulation diagram of the PM-PCF.

800-1700 nm. A 10/125 $\mu \mathrm{m}$ PM-CIR (fast axis blocked) is used as a polarizer to improve the pump PER to $25 \mathrm{~dB}$, as well as an isolator to protect the seed from backward propagating light. The PM-PCF (NKT Photonics LMAPM-5) has a core diameter of $4.4 \mu \mathrm{m}$, an air-hole pitch of $3.32 \mu \mathrm{m}$ and an air-hole diameter of $1.36 \mu \mathrm{m}$, as shown in the inset of Figure 2, and is utilized as the main nonlinear material to generate a linearly polarized SC. The air-topitch ratio is 0.41 , less than 0.45 , indicating that the PMPCF has the property of maintaining a single mode structure at the operating wavelengths ${ }^{[29]}$. Two stress-application areas create stress on the silica core and induce stress birefringence, resulting in maintaining the polarization state when light propagates along the principal axes in the PMPCFs. The birefringence is $1.5 \times 10^{-4}$ at $1064 \mathrm{~nm}$ for the fiber. The corresponding polarization beat length and attenuation parameters at $1064 \mathrm{~nm}$ are about $7 \mathrm{~mm}$ and less than $7 \mathrm{~dB} / \mathrm{km}$, respectively.

To build an all-fiber structure for compactness and robust operation, the pump source and the PM-PCF are fusion spliced. However, owing to the severe mode field mismatch induced by inconsistency in the core size between the pigtail fiber of the PM-CIR and the PM-PCF, a $0.2 \mathrm{~m}$ long 6/125 $\mu \mathrm{m}$ PM-GDF is utilized as a transition between them. Developing a splicing technique for PCFs can be challenging and require special attention to retain good optical properties at the splicing point. By optimizing the parameters of the fusion splicer, such as discharge intensity and discharge position, two polarization-maintaining splicing joints between the PM-CIR, the PM-GDF and PMPCFs are achieved, with transmission rates of $87 \%$ and $90 \%$, respectively. Also, we make an angle cleaved end cap to eliminate back reflection and protect the PM-PCF from dust, humidity and other contaminants. The output SC spectra are recorded by optical spectrum analyzers, in the range $350-2400 \mathrm{~nm}$, with long-pass filters used to eliminate the effect of high-order diffraction during the measurement. 

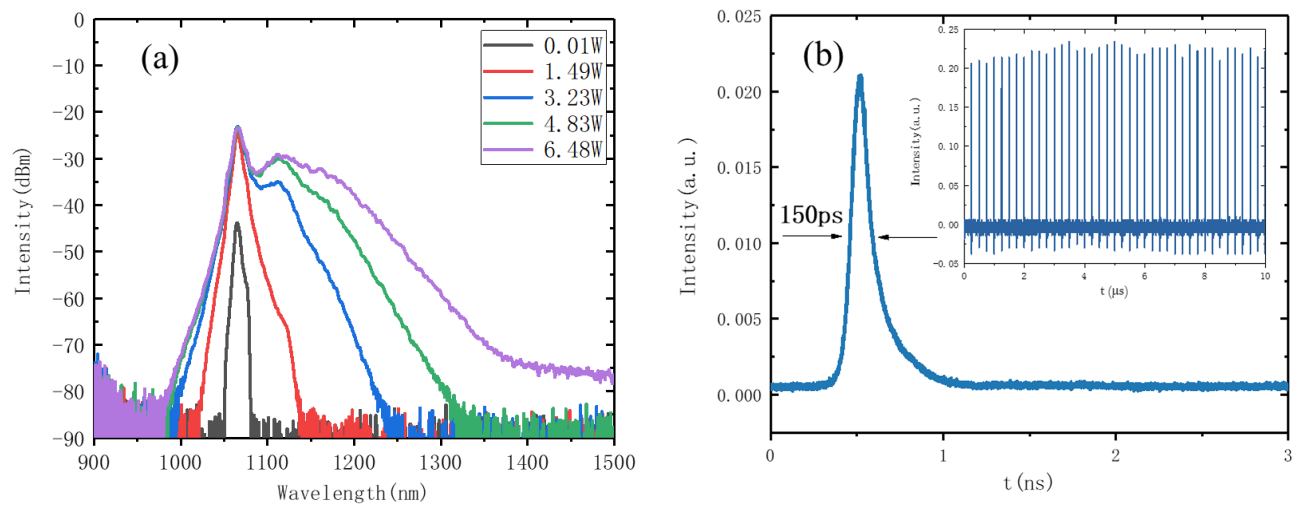

Figure 3. (a) Spectral evolution of the pump source. (b) Single pulse shape and (inset) pulse train shape of the pump source.
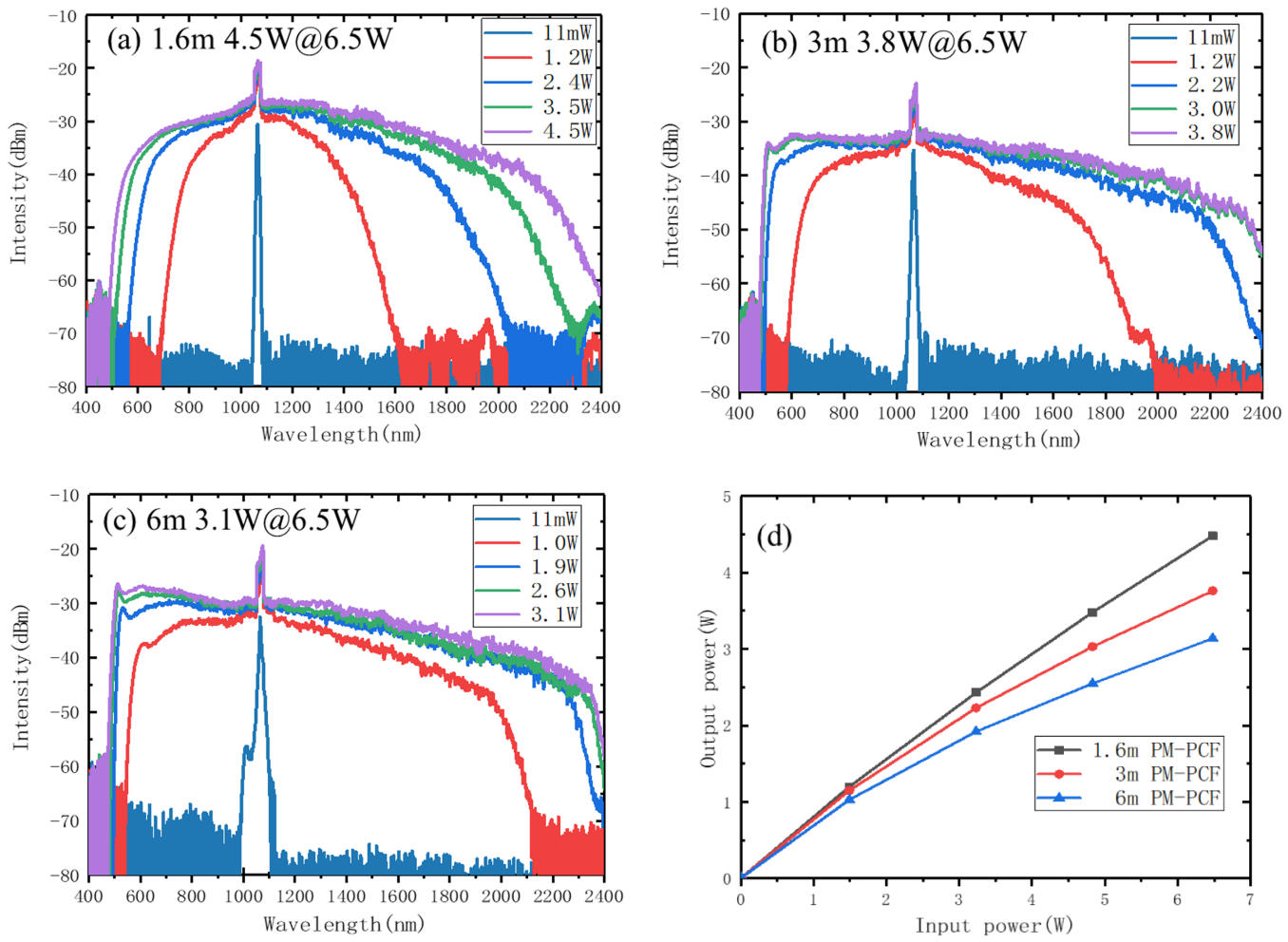

Figure 4. SC generated in different lengths of PM-PCF: (a) $1.6 \mathrm{~m}$ long PM-PCF, (b) $3 \mathrm{~m}$ long PM-PCF and (c) $6 \mathrm{~m}$ long PM-PCF. (d) SC output power versus the pump power.

\section{Results and discussion}

\subsection{Effect of PCF length on SC generation}

Numerical simulation indicates that the ZDW of the slow axis is $1070 \mathrm{~nm}$, as shown in Figure 2. The center wavelength of the pump source is $1064 \mathrm{~nm}$, which is located in the normal group velocity dispersion (GVD) regime of the PM-PCF and relatively near the ZDW. The spectrum of the pump source at different powers before entering the PM-PCF is shown in Figure 3(a). The maximal average pump power is $6.48 \mathrm{~W}$, with a pulse duration of 150 ps at a repetition rate of $4 \mathrm{MHz}$ (Figure 3(b)). The corresponding peak power and pulse energy are calculated to be $10.8 \mathrm{~kW}$ and $1.7 \mu \mathrm{J}$, respectively.

Three different lengths of PM-PCFs are utilized to study the influence of fiber length on SC generation, including $1.6 \mathrm{~m}, 3 \mathrm{~m}$ and $6 \mathrm{~m}$. The splicing alignment angle is set at $0^{\circ}$ to keep the pump light propagating parallel along the slow axis. Figure 4 illustrates the SC evolution with pump power in different PM-PCF lengths. Since the pump wavelength is located in the normal GVD regime and rel- 
atively close to the ZDW of the PM-PCF at low pump power less than $1.5 \mathrm{~W}$ (red curve in Figure 3(a)), the phasematching condition required by four wave mixing (FWM) can be readily satisfied ${ }^{[30,31]}$. From Figure 4(a), we can see that the spectrum is broadened almost symmetrically relative to the pump wavelength in the $1.6 \mathrm{~m}$ long PM-PCF when pumped at low power (the red curve). Symmetry appears in the spectrum because of the greater gain of the parametric process relative to Raman scattering. In addition, the Raman spectrum of the pump light, which covers the anomalous dispersion region of the fiber, is sufficient to stimulate the nonlinear effect related to solitons at a high power level (green and purple curves in Figure 3(a)). The following is composed mainly of Raman soliton self-frequency shift (SSFS) and modulation instability (MI). The SSFS broadens the spectrum to the longer wavelength range over $2400 \mathrm{~nm}$, reaching the inherent loss region of the silica fiber. At the same time, dispersive wave generation broadens the spectrum to short wavelengths, forming the asymmetrical spectral shape, as the green and purple curves in Figure 4 show. In each length of PM-PCF, the SC extends over a bandwidth of $1900 \mathrm{~nm}$ (from $480 \mathrm{~nm}$ to over $2400 \mathrm{~nm}$ ) at the highest pump power. In particular, the source exhibits a flat spectrum from $480 \mathrm{~nm}$ to $2100 \mathrm{~nm}$ at the $-10 \mathrm{~dB}$ level, except for the residual pump peak for the $3 \mathrm{~m}$ long PM-PCF, as shown in Figure 4(b).

Figure 4(d) shows the average SC output power versus the corresponding PM-PCF average input power, with slope efficiencies of $69 \%, 58 \%$ and $48 \%$, respectively, for the $1.6 \mathrm{~m}, 3 \mathrm{~m}$ and $6 \mathrm{~m}$ long PM-PCFs. A maximal output power of $4.5 \mathrm{~W}$ is achieved from the $1.6 \mathrm{~m}$ long PM-PCF. Because the accumulation of nonlinear effects depends on the length of the nonlinear fiber, the transformation of pump light to the SC is insufficient in the $1.6 \mathrm{~m}$ long PM-PCF for this case, although it can achieve a higher power and conversion efficiency. In contrast, the $6 \mathrm{~m}$ long PM-PCF (Figure 4(c)) generates a broader SC than the $3 \mathrm{~m}$ and $1.6 \mathrm{~m}$ PM-PCFs when pumped under the same conditions, as shown in Figures 4(a)-4(c). Due to the inherent absorption loss of silica-based fibers, the intensity around the $2400 \mathrm{~nm}$ long-wavelength edge for the $6 \mathrm{~m}$ long PM-PCF decreases, while more dispersion waves are captured in the shortwavelength edge compared to the spectrum generated in the $3 \mathrm{~m}$ long PM-PCF, as can be seen from Figures 4(b) and 4(c). Taking the slope efficiency, the output power and the spectral broadening into account, the $3 \mathrm{~m}$ long PM-PCF is considered as the best of the three choices $(1.6 \mathrm{~m}, 3 \mathrm{~m}$ and $6 \mathrm{~m}$ in the experiment) for linearly polarized SC generation in this case. Note that the optimal fiber length for SC generation is expected to be related to other conditions, such as the pump peak power and pump wavelengths. With increasing pump power, we observe no obvious temperature increase of the PM-PCF, indicating the good stability and reliability of the linearly polarized SC source and the possibility of further increasing the output power of the linearly polarized SC.
The PER of the SC ranging from $800 \mathrm{~nm}$ to $1500 \mathrm{~nm}$, pumped at low average power, is over $20 \mathrm{~dB}$, as measured by an extinction ratio meter. Also, by using filters and halfwave plates, the PERs of the SC at the high pump power level at single wavelengths, including $532 \mathrm{~nm}, 1064 \mathrm{~nm}$ and $1550 \mathrm{~nm}$, are all measured to be greater than $20 \mathrm{~dB}$. These results indicate that the generated SC preserves the polarization state of the incident pump light.

Regarding the coherence property of the SC, since the PMPCF can maintain the single-mode property at the operating wavelengths, the spatial coherence is expected to be good. However, the temporal coherence is expected to be bad, due to the MI effect.

\subsection{Effect of the polarization state of the pump on SC generation}

3.2.1. Simulation of the SC generated in the slow and fast axes

Due to birefringence in the fiber core induced by the two symmetric stress rods, the group velocity dispersion properties of the two orthogonal axes are different, resulting in a different SC generation process. Through geometric modeling using the COMSOL Multiphysics software, we obtained the slow axis refractive index distribution. According to the nominal birefringence at $1064 \mathrm{~nm}$, we add the birefringence to the index profile. The result can be considered approximately as the fast axis refractive index, because the magnitude of the birefringence is $10^{-4}$, which is relatively small compared to the inherent refractive index. Therefore, fiber parameters, including the effective refractive index and high-order dispersion terms of the two orthogonal polarization modes under different wavelengths, can be calculated. Figure 5(a) gives the simulated dispersion curves, which show that the ZDW is $1070 \mathrm{~nm}$ for the slow axis and $1075 \mathrm{~nm}$ for the fast axis, indicating a $5 \mathrm{~nm}$ interval between the two orthogonal axes.

Then, we use the generalized Schrödinger equation (GNLSE) in the model, where propagation is assumed along only one axis of the fiber in the fundamental mode. The equation is solved by the split-step Fourier transform method. We analyze the SC spectrum pumped at $1064 \mathrm{~nm}$ in a piece of $1 \mathrm{~m}$ long PM-PCF. The peak power and pulse duration are $10.8 \mathrm{~kW}$ and $150 \mathrm{ps}$, consistent with the experiment. Figure 5(b) is the spectral evolution along the fiber length when pump light propagates along the slow axis. Two side bands are generated initially at $800 \mathrm{~nm}$ and $1500 \mathrm{~nm}$, which is a typical phenomenon of FWM in the normal-dispersion region. Meanwhile, the SC generated in the slow axis, as shown in Figure 6(a), broadens to a short-wavelength edge of $580 \mathrm{~nm}$, while a $600 \mathrm{~nm}$ shortwavelength edge is obtained in the fast axis, as shown in Figure 6(b), indicating a $20 \mathrm{~nm}$ blue-extended spectrum for the slow axis compared to the fast axis. The long-wavelength 

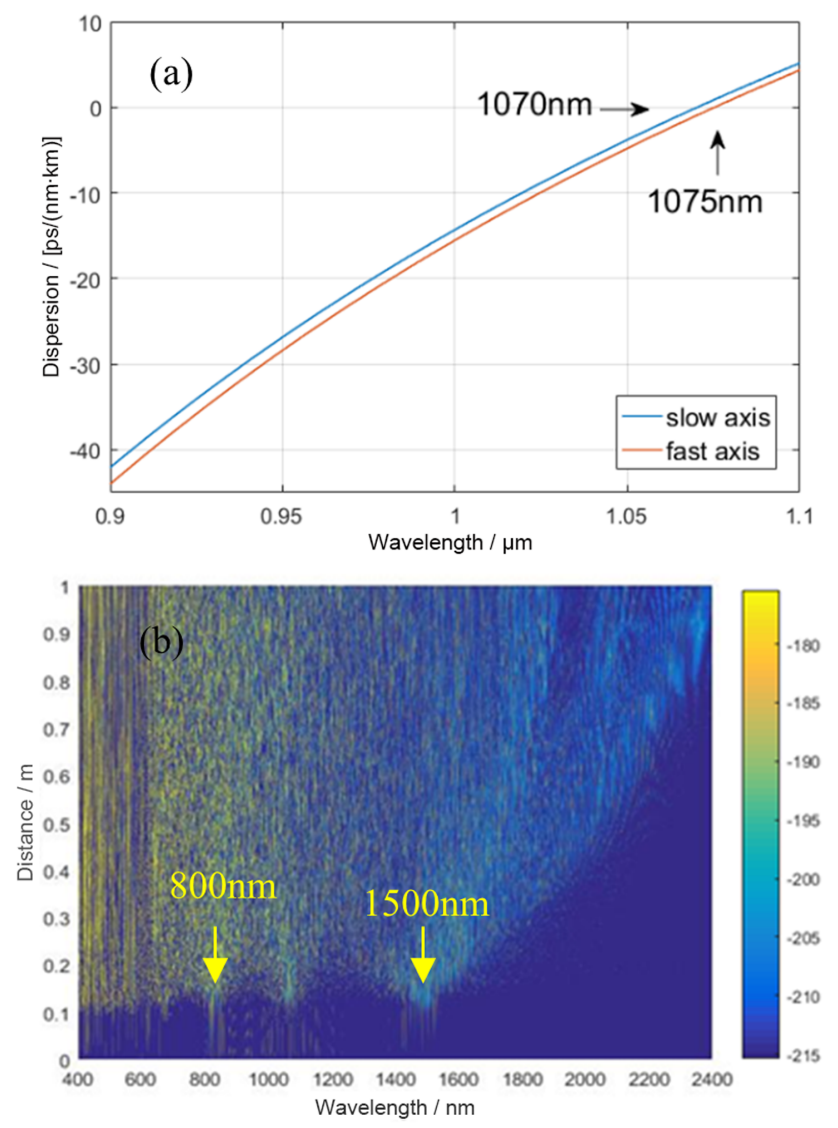

Figure 5. (a) Simulated dispersion curves for the fast axis and slow axis. (b) Spectral evolution along the fiber length for the slow axis.

edge of the SC is up to $2400 \mathrm{~nm}$ in both principal axes. Owing to the different dispersion properties of the two axes, the long-wavelength edge near $2400 \mathrm{~nm}$ in the fast axis has a significantly sharper decrease compared to that of the slow axis. The simulation results indicate that a broader SC can be generated in this PM-PCF when pumped in the slow axis.

3.2.2. Experimental $\mathrm{SC}$ generation in the slow and fast axes and at $45^{\circ}$ to the slow axis

According to the previous experimental results, $3 \mathrm{~m}$ long PM-PCF is utilized as the nonlinear medium for SC generation. An extra splicing point on the $6 / 125 \mu \mathrm{m}$ PM-GDF is made to adjust the polarization direction of the pump light into the PM-PCF. The extra splicing angle is set at $0^{\circ}$ (slow axis), $90^{\circ}$ (fast axis) and $45^{\circ}$ to the slow axis, respectively.

As shown in Figure 7(a), when pumped by $10.8 \mathrm{~kW}$ peak power, we notice that both the short- and long-wavelength edges are obviously different, as expected in the simulation. The cutoff short-wavelength boundaries are $477 \mathrm{~nm}, 498 \mathrm{~nm}$ and $508 \mathrm{~nm}$ for the slow axis, the fast axis and at $45^{\circ}$ to the slow axis. This can be explained as follows: when pump light propagates parallel to the principal axis, only one of the orthogonal polarization modes is stimulated. Because the pump wavelength is closer to the ZDW of the slow axis,
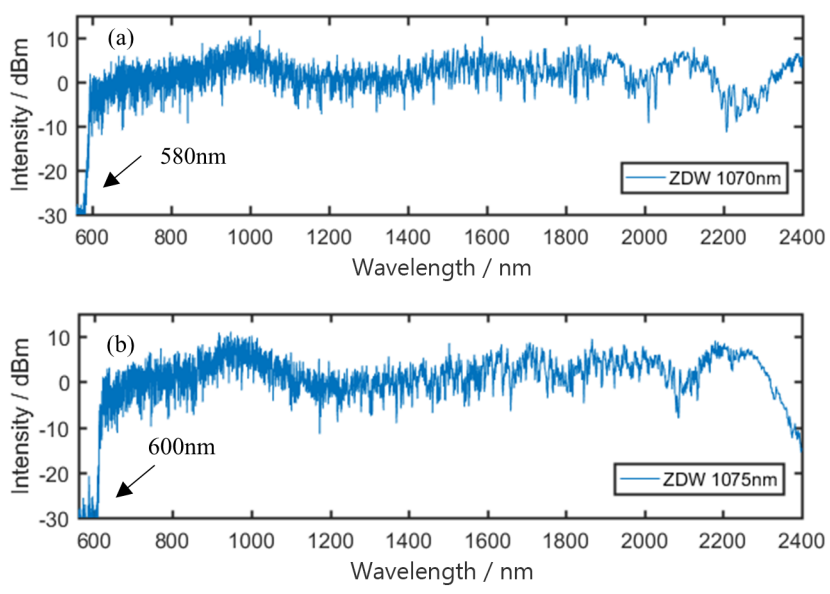

Figure 6. Simulated SC spectra for the (a) slow axis and (b) fast axis.

more dispersion waves are captured by redshift solitons. However, the pulse energy is split into the two principal axes when light propagates along a line at $45^{\circ}$ to slow axis, resulting in only half of the peak power excited in each of the two polarization modes. So the spectrum is limited to 500-2300 nm. Corresponding efficiency curves are plotted in Figure 7(b). The red curve shows that light propagates at $45^{\circ}$ to the slow axis, which exhibits the maximum output power, but the corresponding spectral broadening is the narrowest. In contrast, when light is transmitted along the slow axis, although the output power is the lowest, the spectrum exhibits the maximum bandwidth.

It should be noted that there is a clear difference in the spectral shape and exact wavelength edges between the simulation and the experiment, probably due to the simplification of the model used, such as the fiber parameters and the single pump wavelength, which is quite different from the real pump laser shown in Figure 3(a). However, the measured and simulated spectra of the SC generated in the two axes are in good agreement in terms of broadening tendency.

\section{Conclusion}

In conclusion, we report an all-fiber, high-power linearly polarized SC source based on PM-PCF. The PM-PCF is pumped by a picosecond pulsed laser, operating at $1064 \mathrm{~nm}$ with a pulse duration and repetition rate of $150 \mathrm{ps}$ and $4 \mathrm{MHz}$, respectively. When the pump peak power reaches $10.8 \mathrm{~kW}$, a flat SC with a broad bandwidth over $1900 \mathrm{~nm}$, from $480 \mathrm{~nm}$ to more than $2400 \mathrm{~nm}$, is achieved with an output power of $3.8 \mathrm{~W}$. The PER is measured to be greater than $20 \mathrm{~dB}$ at selected sample wavelength points $(532 \mathrm{~nm}$, $1064 \mathrm{~nm}$ and $1550 \mathrm{~nm}$ ) at the highest pump power level and greater than $20 \mathrm{~dB}$ at all wavelengths from $800 \mathrm{~nm}$ to $1500 \mathrm{~nm}$ at the low pump power level. Through comparison 

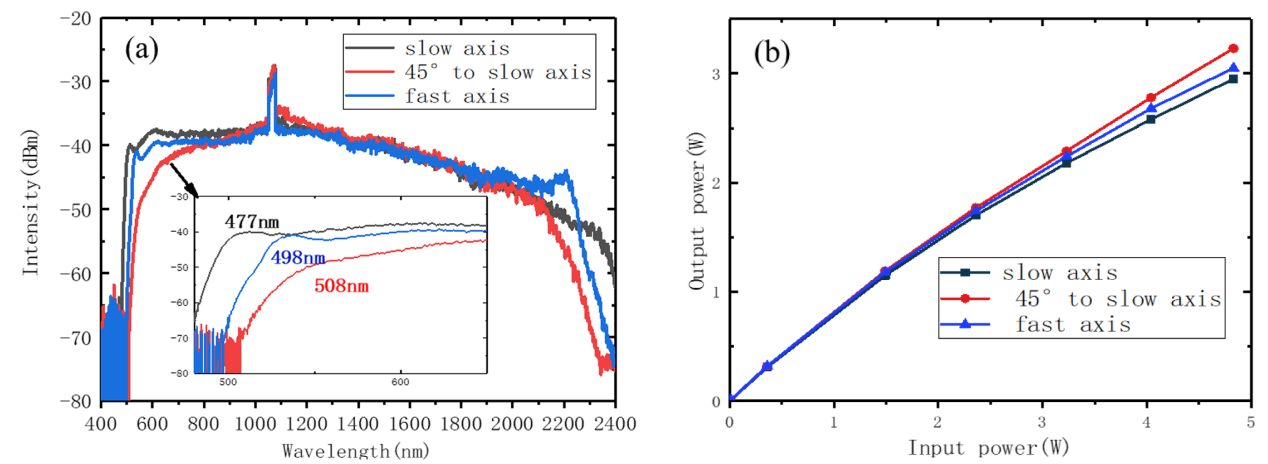

Figure 7. (a) Spectra and (b) output power versus pump power along slow axis, fast axis and $45^{\circ}$ to slow axis.

between $1.6 \mathrm{~m}, 3 \mathrm{~m}$, and $6 \mathrm{~m}$ PM-PCFs, we find that $3 \mathrm{~m}$ long PM-PCF is an appropriate length for $\mathrm{SC}$ generation in our experiment. We also theoretically analyze the SC property when the pump pulses are polarized along different directions of the PM-PCF, and experimentally verify that the spectrum is broader along the slow axis in the PM-PCF.

\section{References}

1. T. Hakala, J. Suomalainen, S. Kaasalainen, and Y. Chen, Opt. Express 20, 7119 (2012).

2. K. Hansen, P. Klarskov, and P. E. Andersen, Opt. Express 19, 26672 (2011).

3. M. Tsuzuki, L. Jin, M. Yamanaka, V. Sonnenchein, H. Tomita, A. Sato, T. Ohara, Y. Sakakibara, E. Omoda, H. Kataura, T. Iguchi, and N. Nishizawa, Photon. Res. 4, 313 (2016).

4. D. M. Owen, E. Auksorius, H. B. Manning, C. B. Talbot, P. A. A. D. Beule, and C. Dunsby, Opt. Lett. 32, 3408 (2007).

5. H. Tu and S. A. Boppart, Opt. Express 17, 9858 (2015).

6. P. Wang, T. Lee, M. Ding, Z. Lian, X. Feng, Y. Ma, L. Bo, Q. Wu, Y. Semenova, W. Loh, G. Farrell, and G. Brambilla, J. Lightwave Technol. 32, 40 (2014).

7. G. Genty, T. Ritari, and H. Ludvigsen, Opt. Express 13, 8625 (2005).

8. C. Stphane, A. H. L. Chau, R. Leonhardt, J. D. Harvey, J. C. Knight, W. J. Wadsworth, and P. S. J. Russell, Opt. Lett. 26, 1356 (2001).

9. X. Qi, S. P. Chen, A. J. Jin, T. Liu, and J. Hou, Opt. Eng. 54, 066102 (2015).

10. P. Jamatia, T. S. Saini, A. Kumar, and R. K. Sinha, Appl. Opt. 55, 6775 (2016).

11. A. Kumar, R. K. Sinha, and T. S. Saini, J. Lightwave Technol. 33, 3914 (2015).

12. A. G. N. Chaitanya, S. T. Singh, K. Ajeet, and S. R. Kumar, Appl. Opt. 55, 10138 (2016).
13. T. S. Saini, N. P. T. Hoa, T. H. Tuan, X. Luo, T. Suzuki, and Y. Ohishi, Appl. Opt. 58, 415 (2019).

14. T. S. Saini, A. Kumar, and R. K. Sinha, in Frontiers in Optics (Optical Society of America, 2014), paper FW1D.4.

15. D. Dobrakowski, A. Rampur, G. Stępniewski, A. Anuszkiewicz, J. Lisowska, D. Pysz, R. Kasztelanic, and M. Klimczak, J. Opt. 21, 015504 (2018).

16. T. Hakala, J. Suomalainen, S. Kaasalainen, and Y. Chen, Opt. Express 20, 7119 (2012).

17. P. Blandin, F. Druon, M. Hanna, S. Lvquefort, C. Lesvigne, and V. Couderc, Opt. Express 16, 18844 (2008).

18. B. Zhang, A. Jin, P. Ma, S. Chen, and J. Hou, Opt. Express 23, 28683 (2015)

19. S. Coen, A. H. Chau, R. Leonhardt, J. D. Harvey, J. C. Knight, W. J. Wadsworth, and P. St J. Russell, Opt. Lett. 26, 1356 (2001).

20. X. Qi, S. Chen, Z. Li, T. Liu, Y. Ou, N. Wang, and J. Hou, Opt. Lett. 43, 1019 (2018).

21. C. Xiong and W. J. Wadsworth, Opt. Express 16, 2438 (2008).

22. Y. Yu, S. Ruan, C. Du, J. Zhao, and J. Zeng, Proceedings of SPIE - The International Society for Optical Engineering (2006), p. 6025.

23. Z. Zhu and T. G. Brown, Opt. Express 12, 791 (2004).

24. P. Yan, J. Shu, S. Ruan, J. Zhao, J. Zhao, C. Du, C. Guo, H. Wei, and J. Luo, Opt. Express 19, 4985 (2011).

25. H. G. Choi, C. S. Kee, J. H. Sun, T. J. Yu, D. K. Ko, and J. Lee, Phys. Rev. A 77, 156 (2008).

26. X. Y. Wang, S. G. Li, Y. Han, Y. Du, C. M. Xia, and L. T. Hou, Sci. China Phys. Mech. Astron. 55, 199 (2012).

27. M. Lehtonen, G. Genty, H. Ludvigsen, and M. Kaivola, Appl. Phys. Lett. 82, 2197 (2003).

28. K. Tarnowski, T. Martynkien, P. Mergo, K. Poturaj, A. Anuszkiewicz, P. Bjot, and P. Krzysztof, Opt. Express 25, 27452 (2017)

29. T. A. Birks, J. C. Knight, and P. S. J. Russell, Opt. Lett. 22, 961 (1997).

30. J. M. Dudley, G. Genty, and S. Coen, Rev. Mod. Phys. 8, 1135 (2006).

31. S. Zhang, X. Yang, F. L, Y. Gong, and X. Meng, Opt. Eng. 47, 075005 (2008). 